\title{
Liposuktion bei Lipödem - Entscheidung des G-BA
}

Im Juni erhielten wir die Aufforderung, gegenüber dem Gemeinsamen Bundesausschuss (G-BA) eine Stellungnahme zur geplanten Kostenübernahme bei Lipödem im Stadium III zu verfassen. Trotz der Kürze der Zeit wurde von der hierzu eingesetzten Taskforce Lipödem/Liposuktion der Deutschen Gesellschaft für Phlebologie gemeinsam mit der Deutschen Gesellschaft für Lymphologie durch Frau Dr. Gabriele Faerber, Dr. Tobias Hirsch, Dr. Karsten Hartmann, Dr. Tobias Bertsch und Prof. Dr. Markus Stücker eine Stellungnahme verfasst und termingerecht abgegeben.

Im August waren Frau Dr. Anya Miller und Dr. Erika Mendoza für die DGP bei der Anhörung vor dem gemeinsamen Bundesausschuss in Berlin. Die Stellungnahme der DGA (Prof. Schellong) und der DGP waren fast deckungsgleich: nicht das Gewicht, sondern den BMI als Begrenzung zur Zulassung zu nehmen - besser noch den Waist to Height ratio. Dabei hatten wir einen BMI von < 32 vorgeschlagen, sowie bei der Indikation das 4-Augen Prinzip und die Diagnosesicherung durch einen nicht chirurgisch tätigen Kollegen. Die Vorsitzende gab gleich zu erkennen, dass der Ausschuss selber lieber erst das Ergebnis der vom Ausschuss in Auftrag gegebenen Studie zum Nachweis der Wirksamkeit der Liposuktion abgewartet hätte, aber dass diese neue Initiative direkt dem ministerialen Willen entsprungen und alternativlos sei.

Die Entscheidung beim GBA wurde am 19.09.2019 getroffen. Ergebnis: Der gemeinsame Bundesausschuss (G-BA) hat die Kostenübernahme für die Liposuktion ausschließlich im Stadium III bis zu einem $B M I$ von $40 \mathrm{~kg} / \mathrm{m}^{2}$ unter Auflagen geneh-

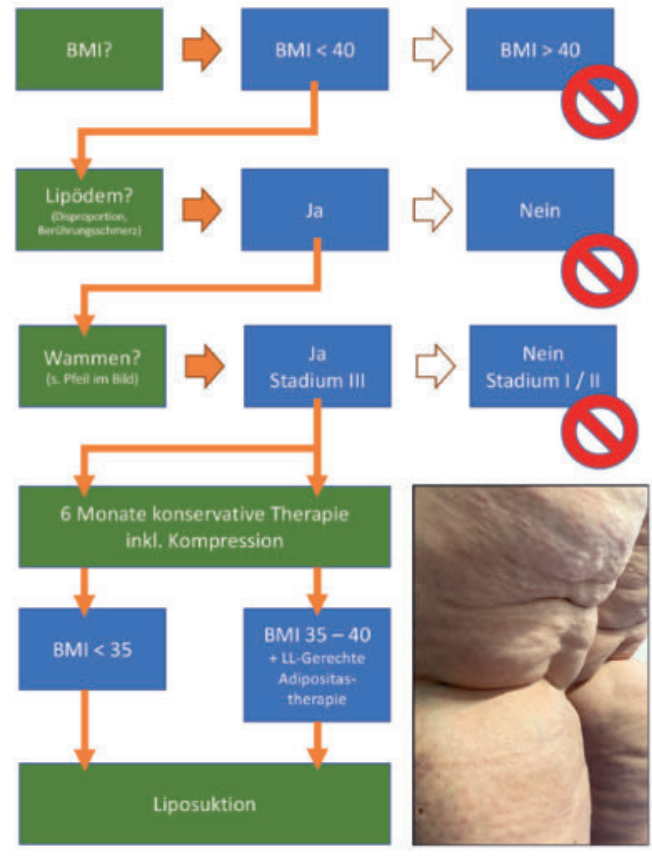

Abb. 1 Flow-Chart zur Pressemitteilung „Liposuktion“ - um auf einem Blick den Entscheidungsweg für die Kollegen aufzuzeigen. (Quelle Foto: Arrien $\mathrm{GmbH}$ mit freundlicher Genehmigung).

migt (s. - Abb. 1). Da es sich beim Lipödem im Stadium III ohne begleitende Adipositas um ein relativ seltenes Krankheitsbild handelt, empfiehlt die Deutsche Gesellschaft für Phlebologie die zusätzliche Dokumentation der WHtR als Entscheidungsgrundlage. Leider ist die vom G-BA geplante Studie zur Wirksamkeit der Liposuktion bei Lipödem noch nicht abgeschlossen, daher wissen wir heute nicht, ob diese Maßnahme für die Patientinnen sinnvoll ist. Hierzu gibt die DGP zusammen mit der DGL eine Pressemitteilung heraus, die wir hier auch für unsere Leser abdrucken.
Diese Information und weitere Handreichungen für Ihre Praxis stehen auch auf unserer Homepage.

Als DGP hätten wir es sehr begrüßt, wenn die Indikation zum Eingriff - wie von uns gefordert - dem 4-Augen-Prinzip unterläge und nicht vom Operateur selbst gestellt würde. Wir empfehlen konsequent, die 6-monatige dokumentierte Vorsorge, sowie die Nachsorge durch die operierenden Zentren durchführen zu lassen. 


\section{Pressemitteilung der}

Deutschen Gesellschaft für Phlebologie zur Entscheidung des Gemeinsamen Bundesausschusses (G-BA) über die Kostenübernahme der Liposuktion beim Lipödem im Stadium III zu Lasten der gesetzlichen Krankenkassen

Das Lipödem ist eine Erkrankung, die gekennzeichnet ist durch Schmerzen, sowie eine Disproportion zwischen einem schmalen Rumpf und Extremitäten mit vermehrtem, berührungsempfindlichem Unterhautfettgewebe.

Im Stadium I der Erkrankung ist die Haut glatt, im Stadium II wird sie uneben mit deutlicher Dellenbildung. Im Stadium III finden sich Hautlappen und Überhänge, so genannte „Wammen“ (s.>Abb. 1). Die Entwicklung vom Stadium I zum Stadium II oder III korreliert mit der Gewichtszunahme, wobei die Stadieneinteilung lediglich die Zunahme der Gewebeveränderungen beschreibt und keinerlei Aussage über die Beschwerdeintensität erlaubt. Die Beschwerden nehmen in der Regel durch Gewichtszunahme zu, können sich nach Ernährungsumstellung und Gewichtsreduktion aber auch wieder bessern.

Ab einem BMI über 32 ist kaum mehr zu unterscheiden, ob die Beinform dem Übergewicht oder dem Lipödem geschuldet ist.
Die „Waist-to-Height-Ratio“ (WHtR) kennzeichnet das Verhältnis zwischen Taille und Körpergröße (Taillenumfang in cm/Größe in $\mathrm{cm}$ ) und ermöglicht bei Werten über 0,5 die Feststellung einer Adipositas.

Der gemeinsame Bundesausschuss (G-BA) hat die Kostenübernahme für die Liposuktion ausschließlich im Stadium III bis zu einem BMI von $40 \mathrm{~kg} / \mathrm{m}^{2}$ unter Auflagen genehmigt. Da es sich beim Lipödem im Stadium III ohne begleitende Adipositas um ein relativ seltenes Krankheitsbild handelt, empfiehlt die Deutsche Gesellschaft für Phlebologie die zusätzliche Dokumentation der WHtR als Entscheidungsgrundlage. Leider ist die vom G-BA geplante Studie zur Wirksamkeit der Liposuktion bei Lipödem noch nicht abgeschlossen, daher wissen wir heute nicht, ob diese Maßnahme für die Patientinnen sinnvoll ist.

Auf der Grundlage des G-BA-Beschlusses kann nunmehr Patientinnen, die aufgrund ihres Lipödems im Stadium III eine ausgeprägte Bewegungseinschränkung aufweisen, schneller geholfen werden.

Voraussetzung für die Liposuktion ist allerdings, dass eine sechsmonatige konservative Therapiephase nicht zum Erfolg geführt hat. Diese beinhaltet das konsequente Tragen flachgestrickter Kompressionshilfsmittel, Bewegungstherapie und im Falle der begleitenden Adipositas (BMI $>30 \mathrm{~kg} / \mathrm{m}^{2}$ ) eine Ernährungsumstellung mit dem Ziel, über 6 Monate mindestens eine Gewichtsstabilität nachzuweisen.
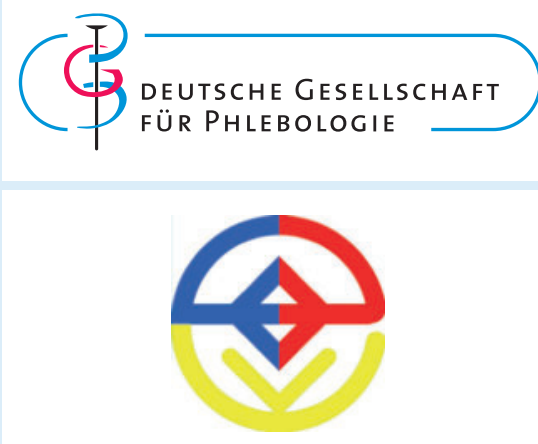

Bei einem BMI zwischen 35 und $40 \mathrm{~kg} / \mathrm{m}^{2}$ muss zusätzlich eine leitliniengerechte Adipositastherapie eingeleitet werden, da ein nachhaltiger Erfolg der Liposuktion ohne Gewichtsreduktion nicht zu erwarten ist. Bei einem BMI über $40 \mathrm{~kg} / \mathrm{m}^{2}$ wird keine Liposuktion mehr erstattet, da hier die Adipositas im Vordergrund steht und entsprechend behandelt werden muss.

Die Indikation zur Liposuktion können laut G-BA Beschluss Fachärzte für Plastische Chirurgie und chirurgisch tätige Fachärzte stellen. Die Prüfung der Indikationskriterien, sowie die Verordnung und Organisation der nach der Liposuktion erforderlichen Behandlung in Form von Lymphdrainagen und Kompression ist Bestandteil der ärztlichen Leistung des jeweiligen Operateurs.
Dr. med. Gabriele Färber,
Dr. med. Anya Miller (DGL)
Dr. med. Tobias Hirsch
Dr. med. Karsten Hartmann
Dr. med. Erika Mendoza
Prof. Dr. med. Markus Stücker 